\title{
Preserving a Rare Type of Variant Right Hepatic Artery Combines Surgical Radicality and Intact Liver Perfusion during Pancreatectomy
}

\author{
Ilgar Aghalarov ${ }^{\mathrm{a}} \quad$ Theodor Lutz $^{\mathrm{b}} \quad$ Waldemar Uhl $^{\mathrm{a}} \quad$ Orlin Belyaev $^{\mathrm{a}}$ \\ ${ }^{a}$ Department of General and Visceral Surgery, St. Josef Hospital Bochum, Ruhr University Bochum, \\ Bochum, Germany; ${ }^{b}$ Department of Radiology and Nuclear Medicine, St. Josef Hospital Bochum, Ruhr University \\ Bochum, Bochum, Germany
}

\begin{abstract}
Keywords
Pancreatectomy · Hepatic artery · Variant anatomy · Michels' classification · Pancreatic cancer
\end{abstract}

\begin{abstract}
An anomalous anatomy of the celiac trunk, and particularly of the right hepatic artery, may have a significant impact on major hepatobiliary and pancreatic surgery. According to some authors, every third patient has an aberrant right hepatic artery. We present a very rare case of replaced right hepatic artery (RRHA) arising from the gastroduodenal artery associated with an accessory left hepatic artery originating from the left gastric artery in a 54-year-old woman with a pancreatic head carcinoma. The patient underwent total pancreatectomy on account of a soft lipomatous pancreas with heterogeneous changes of the pancreatic body and tail. We preserved the RRHA and achieved $\mathrm{R} 0$ resection margins. Preoperative evaluation of CT angiograms, an awareness of any anomalous arterial anatomy of the upper abdomen, and a meticulous surgical technique are the key to performing oncologically radical surgery without threatening the arterial liver supply.

ㄷ 2020 S. Karger AG, Basel
\end{abstract}

\section{Introduction}

Knowledge of any anomalous anatomy of the superior mesenteric artery, celiac trunk, and particularly hepatic arteries is of great importance to prevent inadvertent complications of major hepatobiliary and pancreatic surgery such as pancreatoduodenectomy (PD) [1-5]. An ab- errant hepatic arterial anatomy occurs in approximately $20-75 \%$ of cases, and multiple variations have been described [6-8].

In this paper, we present a very rare case of replaced right hepatic artery (RRHA) arising from the gastroduodenal artery (GDA) associated with an accessory left hepatic artery (ALHA) originating from the left gastric artery (LGA).

\section{Case Report}

A 54-year-old woman with painless progressive jaundice showed a double-duct sign caused by a pancreatic head mass as well as heterogeneous changes of the pancreatic body and tail on a CT scan. Endoscopic ultrasound fine needle aspiration of the pancreatic tumor revealed atypical cells. The common bile duct was stented via endoscopic retrograde cholangiography. The patient was referred to our department for the surgical treatment of suspected pancreatic head cancer. Beside a resectable pancreatic head tumor without metastasis, preoperative high-resolution CT scanning demonstrated an RRHA arising from the GDA as well as an ALHA originating from the LGA (Fig. 1, 2).

Explorative laparotomy was performed with curative intent. Intraoperative inspection showed a resectable tumor without distant metastases or peritoneal carcinomatosis. Intraoperative palpation of the foramen of Winslow confirmed the aberrant RHA. Further dissection revealed that the RRHA arose from the intraparenchymal part of the GDA embedded in the anterior face of the pancreatic head and traveling dorsally to the common bile duct in the groove of the superior mesenteric vein behind the pancreatic head. Simultaneously, we detected an ALHA arising from the LGA. Due to the soft and fatty pancreas, as well as heterogeneous changes of the pancreatic body and tail, we decided to perform a total pancreatectomy with splenectomy.

Using meticulous dissection, we successfully spared the RRHA. The GDA was completely dissected along its course. After clamp- 


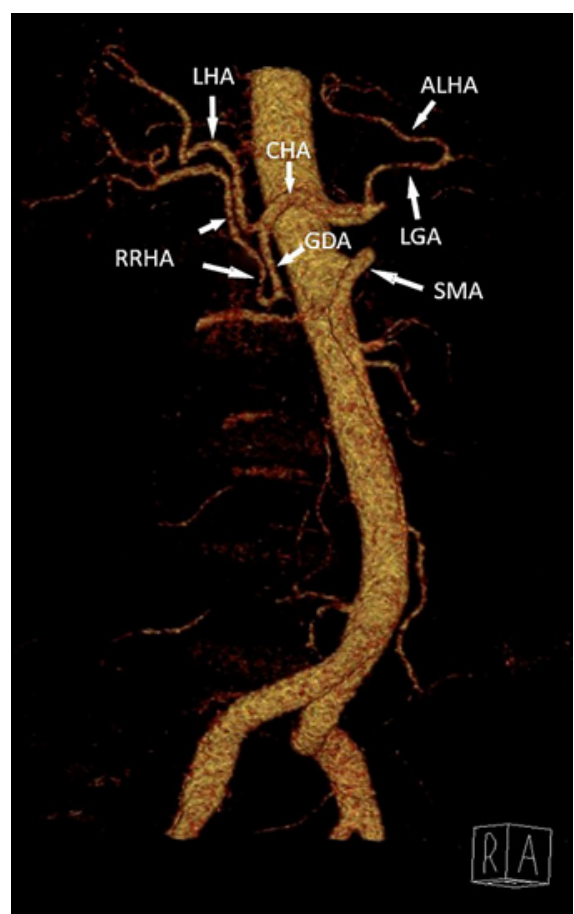

Fig. 1. 3D reconstruction of a computed tomography angiogram (right anterior oblique view) showing an RRHA stemming from the GDA, as well as an ALHA arising from the LGA. RRHA, replaced right hepatic artery; ALHA, accessory left gastric artery; LHA, left hepatic artery; CHA, common hepatic artery; GDA, gastroduodenal artery; LGA, left gastric artery; SMA, superior mesenteric artery.

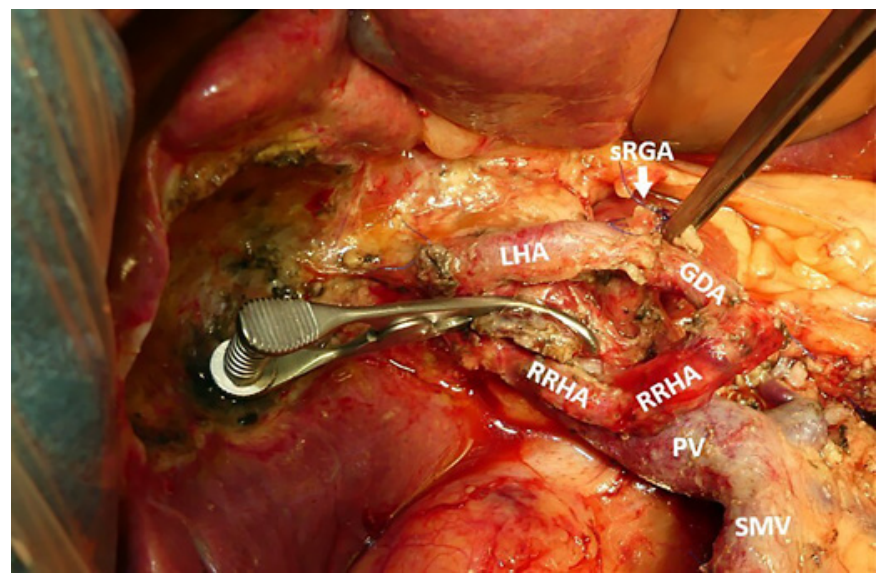

Fig. 3. Intraoperative view after resection of the pancreatic head with sparing of the GDA and RRHA. RRHA, replaced right hepatic artery; LHA, left hepatic artery; GDA, gastroduodenal artery; SMV, superior mesenteric vein; PV, portal vein; sRGA, stump of the right gastric artery.

ing of the GDA distal to the origin of the RRHA, sufficient arterial pulsation of all extrahepatic arteries was confirmed and the GDA was ligated while preserving the RRHA (Fig. 3). The intraoperatively frozen section revealed an R0 margin of the specimen. The diagnosis of pancreatic cancer was verified, and it was staged as pT2pN0M0 G2 adenocarcinoma. Postpancreatectomy hemor-

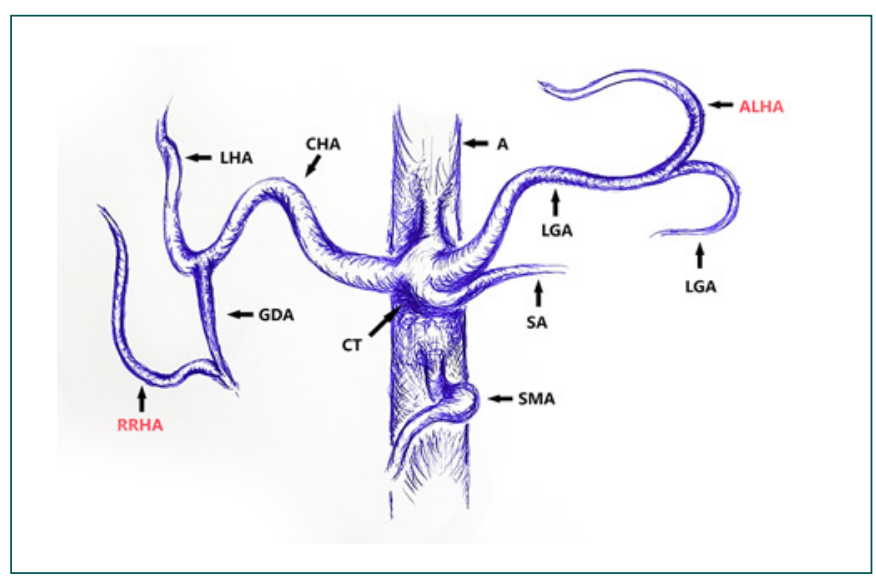

Fig. 2. Sketch of the computed tomography angiogram in Figure 1. RRHA, replaced right hepatic artery; ALHA, accessory left gastric artery; LHA, left hepatic artery; CHA, common hepatic artery; GDA, gastroduodenal artery; LGA, left gastric artery; SMA, superior mesenteric artery; SA, splenic artery; $\mathrm{CT}$, celiac trunk; $\mathrm{A}$, aorta.

rhage grade A occurred on the third day after surgery. CT angiography showed no signs of active bleeding and no intervention was necessary. The patient was discharged on day 21 . According to the multidisciplinary tumor board, a chemotherapy regimen with modified FOLFIRINOX was started 9 weeks postoperatively.

\section{Discussion}

The RHA shows various modifications of the dominant anatomy in 11-29\% of cases [2, 6, 7]. An anomalous arterial anatomy is often revealed just intraoperatively [1]. Intraoperative injury to the RHA increases the rate of complications such as breakdown of hepaticojejunostomy and liver abscess $[1,2]$. Since ligation of the GDA is a critical step of PD, knowing and assuming that the RHA can arise from "anywhere" is of paramount importance. In these cases, the surgeon is confronted with the dilemma between oncologic radicality and surgical safety.

Various strategies have been described to manage an aberrant RHA in major hepatobiliary and pancreatic surgery: (1) resection versus preservation/reconstruction depending on whether the aberrant RHA is accessory or replaced, or whether the tumor infiltrates the vessel; (2) preoperative embolization; (3) if detected intraoperatively, temporary clamping before ligation; and (4) neoadjuvant chemotherapy $[1,9,10]$. An aberrant RHA seems to have no significant influence on the postoperative course and oncological results following PD in cases of early recognition and appropriate management $[3,10]$.

Michels' classification of hepatic arterial variations (from 1966) [6] was modified by Hiatt et al. [7] in 1994. However, there are numerous anatomical variants (1.4$3 \%)$ that cannot be described using Michels' and Hiatt's 
classifications [11]. Numerous classification systems of the hepatic arterial anatomy have been proposed $[8,12$, 13]. In 2014, Kobayashi et al. [13] introduced an easy-touse clinical classification of anatomic variations of the hepatic artery using angiographic images of 1,200 patients. An RRHA originating from the GDA has been described in 11 cases $[4,5,13-15]$.

We presented a very rare case of combination of an RRHA originating from the GDA with an ALHA arising from the LGA. To the best of our knowledge, this type of anomalous arterial anatomy has not been reported yet. Michels type VIII, describing a combination of RRHA and ALHA, denotes an RRHA stemming from the superior mesenteric artery, but not from the GDA. According to Kobayashi's classification, our case could be classified as follows: "I-I, I"; GDA, CHA, LGA (the "I-I" shape resembles the right and left hepatic arteries branching individually without a proper hepatic artery, and the additional "I" represents an accessory artery, in this case to the left of the hepatic artery; "GDA, CHA [common hepatic artery], and LGA" show the origins of each hepatic artery, respectively).

\section{Conclusions}

An aberrant right hepatic artery is the most frequent anomaly of the hepatic arterial tree. To keep it in mind is of paramount importance in hepatobiliary and pancreatic surgery in order to avoid inadvertent severe complications, especially in cases of major open and laparoscopic pancreatic and liver resections. Anomalies of the visceral vessels should also be carefully considered in transplantation surgery, since they may affect liver procurement as well as deceased donor and living donor liver transplants. Preoperative assessment of CT angiograms, as well as a meticulous surgical technique, increases the possibility of preservation of an aberrant hepatic artery in R0 surgery.

\section{Statement of Ethics}

The patient gave her written informed consent to publish the case and the images. In agreement with German legislation, no separate approval by the local ethics committee is necessary in such cases.

\section{Conflict of Interest Statement}

The authors have no conflicts of interest to declare.

\section{Funding Sources}

There was no funding for this study.

\section{Author Contributions}

Ilgar Aghalarov: conception, design, analysis and interpretation of data; drafting and revision of the article; and final approval. Theodor Lutz: analysis and interpretation of data; revision of the article; and final approval. Waldemar Uhl: conception, design, analysis and interpretation of data; revision of the article; and final approval. Orlin Belyaev: conception, design, analysis and interpretation of data; drafting and revision of the article; and final approval.

\section{References}

1 Pallisera A, Morales R, Ramia JM. Tricks and tips in pancreatoduodenectomy. World J Gastrointest Oncol. 2014 Sep;6(9):344-50.

2 Noussios G, Dimitriou I, Chatzis I, Katsourakis A. The Main Anatomic Variations of the Hepatic Artery and Their Importance in Surgical Practice: Review of the Literature. J Clin Med Res. 2017 Apr;9(4):248-52.

3 Németh K, Deshpande R, Máthé Z, Szuák A, Kiss M, Korom C, et al. Extrahepatic arteries of the human liver - anatomical variants and surgical relevancies. Transpl Int. 2015 Oct; 28(10):1216-26.

4 Suzuki T, Nakayasu A, Kawabe K, Takeda H, Honjo I. Surgical significance of anatomic variations of the hepatic artery. Am J Surg. 1971 Oct;122(4):505-12.

5 Chawla A, Rosenthal MH, Clancy TE. Implications of the replaced right hepatic artery originating from the gastroduodenal artery in the setting of a pancreatic head mass. Clin Imaging. 2018 Nov-Dec;52:189-92.

6 Michels NA. Newer anatomy of the liver and its variant blood supply and collateral circula- tion. Am J Surg. 1966 Sep;112(3):337-47.

7 Hiatt JR, Gabbay J, Busuttil RW. Surgical anatomy of the hepatic arteries in 1,000 cases. Ann Surg. 1994 Jul;220(1):50-2.

8 Abdullah SS, Mabrut JY, Garbit V, De La Roche E, Olagne E, Rode A, et al. Anatomical variations of the hepatic artery: study of 932 cases in liver transplantation. Surg Radiol Anat. 2006 Oct;28(5):468-73.

9 El Amrani M, Pruvot FR, Truant S. Management of the right hepatic artery in pancreaticoduodenectomy: a systematic review. J Gastrointest Oncol. 2016 Apr;7(2):298-305.

10 Trofin AM, Vlad N, Zabara M, Rusu-Andriesi D, Bradea C, Vornicu A, et al. Pancreaticoduodenectomy in patients with hepatic artery anatomic variants: tailoring, perioperative care and surgical outcomes. Rev Med Chir Soc Med Nat Iasi. 2016 Oct-Dec;120(4):8749.

11 Sakamoto Y, Fujikawa T, Tanaka A. Successful radical resection of pancreatic head carcinoma in a patient with replaced right hepatic artery originating from posterior inferior pancreaticoduodenal artery: a case report. Surg Case Rep. 2017 Dec;3(1):78.

12 Koops A, Wojciechowski B, Broering DC, Adam G, Krupski-Berdien G. Anatomic variations of the hepatic arteries in 604 selective celiac and superior mesenteric angiographies. Surg Radiol Anat. 2004 Jun;26(3):239-44.

13 Kobayashi S, Otsubo T, Koizumi S, Ariizumi S, Katagiri S, Watanabe T, et al. Anatomic variations of hepatic artery and new clinical classification based on abdominal angiographic images of 1,200 cases. Hepatogastroenterology. 2014 Nov-Dec;61(136):2345-8.

14 Gruttadauria S, Foglieni CS, Doria C, Luca A, Lauro A, Marino IR. The hepatic artery in liver transplantation and surgery: vascular anomalies in 701 cases. Clin Transplant. 2001 Oct;15(5):359-63.

15 Winston CB, Lee NA, Jarnagin WR, Teitcher J, DeMatteo RP, Fong Y, et al. CT angiography for delineation of celiac and superior mesenteric artery variants in patients undergoing hepatobiliary and pancreatic surgery. AJR Am J Roentgenol. 2007 Jul;189(1):W13-9. 